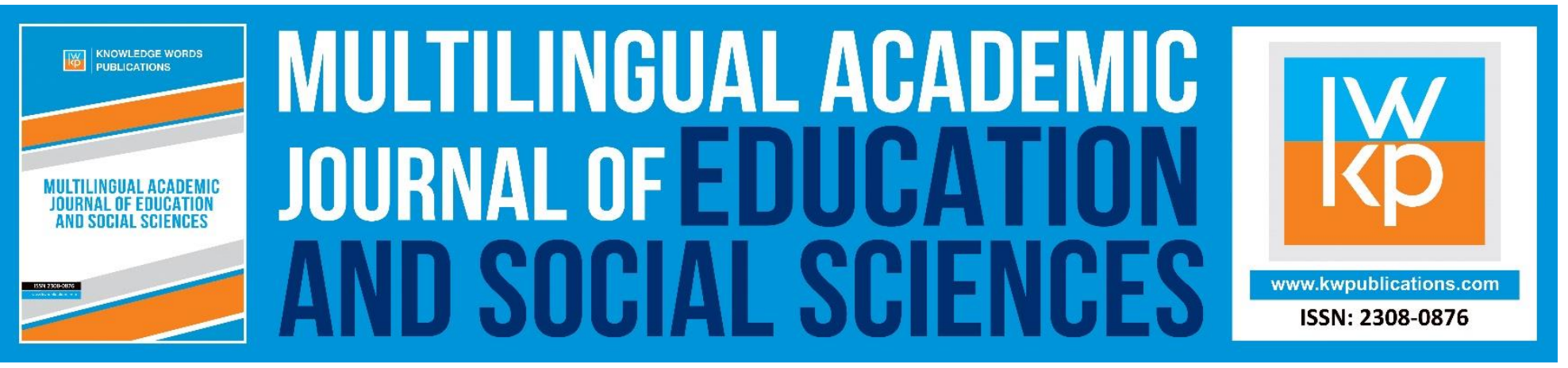

\title{
Newcomers in Greek State Schools: From Classroom Practice to Policy Making
}

\section{Vasilios Zorbas \& Evagelia Papalexatou}

To Link this Article: http://dx.doi.org/10.46886/MAJESS/v5-i1/2429

DOI: 10.46886/MAJESS/v5-i1/2429

Received: 04 June 2017, Revised: 25 July 2017, Accepted: 08 September 2017

Published Online: 16 October 2017

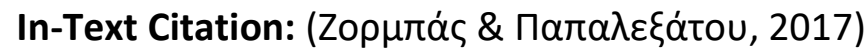

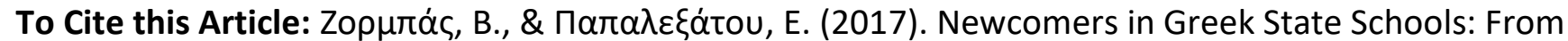
classroom Practice to Policy Making. Multilingual Academic Journal of Education and Social Sciences, 5(1), 57-68.

\section{Copyright: (C) The Authors 2017}

Published by Knowledge Words Publications (www.kwpublications.com)

This article is published under the Creative Commons Attribution (CC BY 4.0) license. Anyone may reproduce, distribute, translate and create derivative works of this article (for both commercial and non-commercial purposes), subject to full attribution to the original publication and authors. The full terms of this license may be seen

at: http://creativecommons.org/licences/by/4.0/legalcode

$$
\text { Vol. 5, No. 1, 2017, Pg. } 57 \text { - } 68
$$

Full Terms \& Conditions of access and use can be found at https://kwpublications.com/pages/detail/publication-ethics 


\title{
Newcomers in Greek State Schools: From Classroom Practice to Policy Making
}

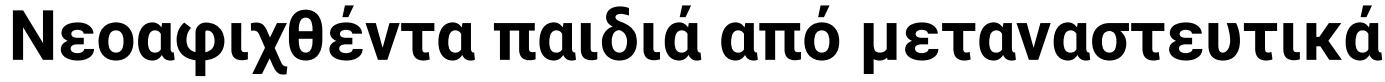

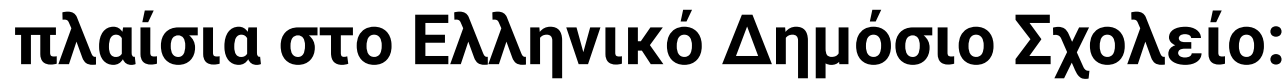

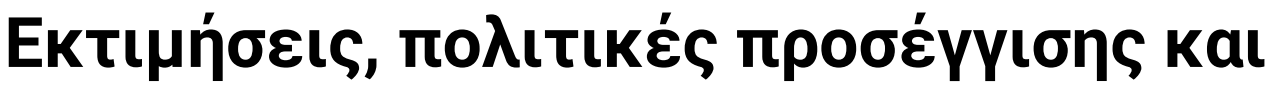

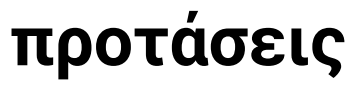

\author{
Vasilios Zorbas ${ }^{1}$ \& Evagelia Papalexatou ${ }^{2}$

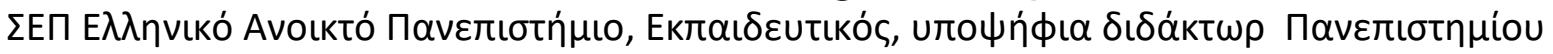 \\ Патри́v.
}

\begin{abstract}
This article centers on the needs of newcomer immigrant students with diverse linguistic and cultural backgrounds in Greek state schools. Setting the discussion against a theoretical background on second language acquisition and bilingualism, we delineate the stages of second language development and provide examples of appropriate instructional strategies per stage which every educator can employ in order to ensure the learners' smooth transition to the target language. The article concludes with a proposal on the integration of immigrant students in Greek state schools.
\end{abstract}

Keywords: Bilingualism, Immigrant Learners, Second Language Acquisition, Teaching Strategies, Education Policy.

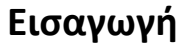

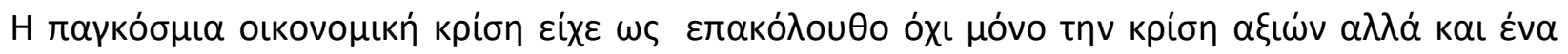

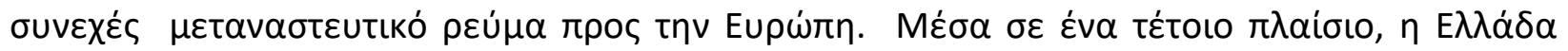
a

\footnotetext{
Bionotes

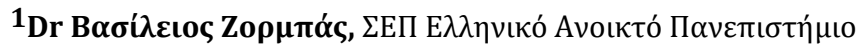

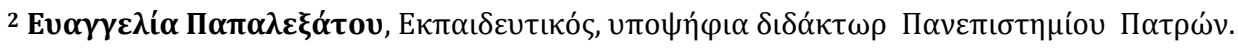


MULTILINGUAL ACADEMIC JOURNAL OF EDUCATION AND SOCIAL SCIENCES

Vol. 5 No. 1, 2017, E-ISSN: 2308-0876 @ 2017 KWP

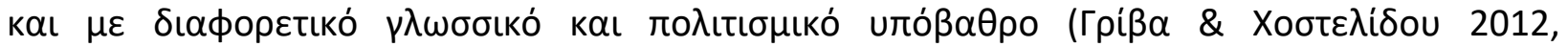

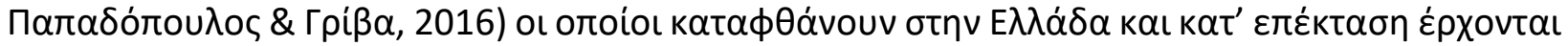

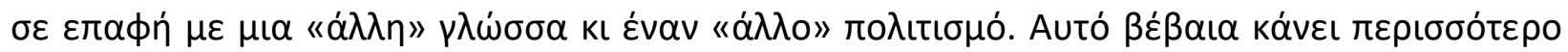

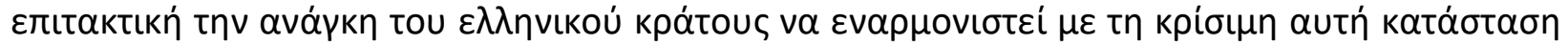

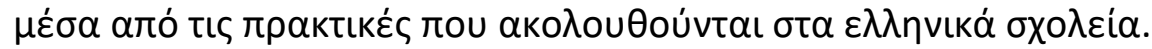

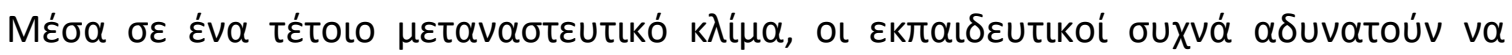

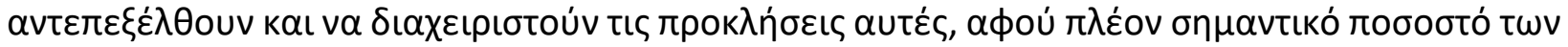

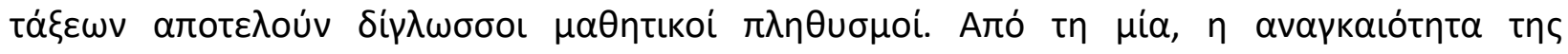

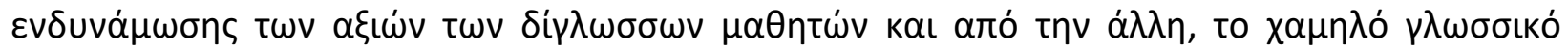

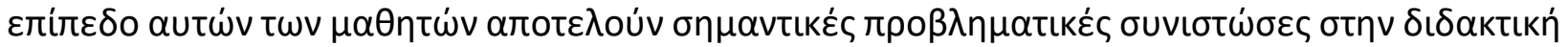

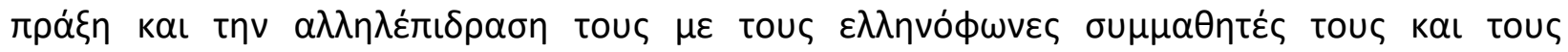

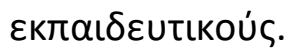

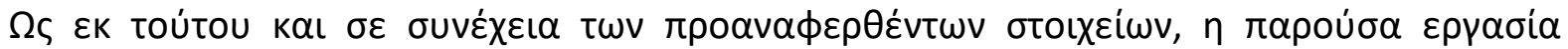

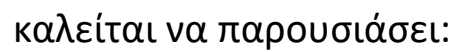

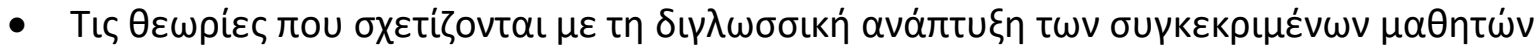

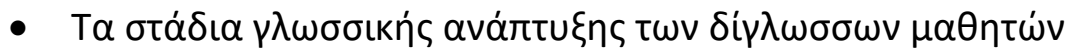

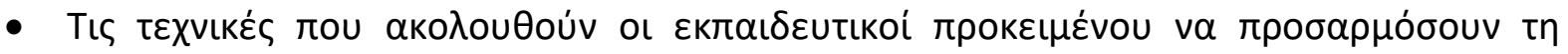

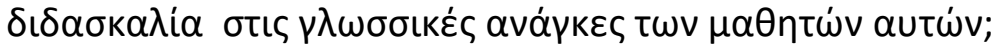

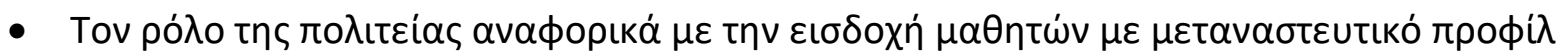

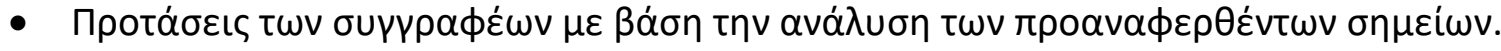

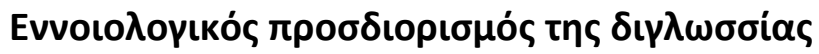

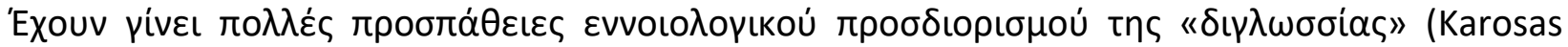

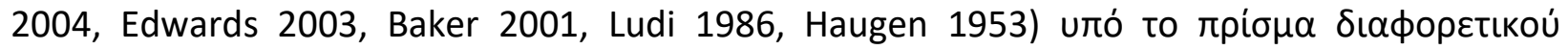

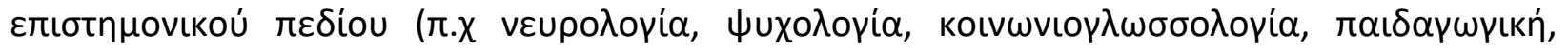

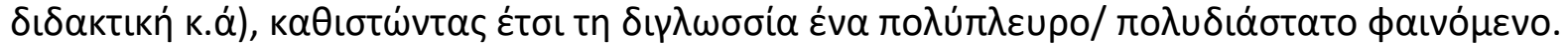

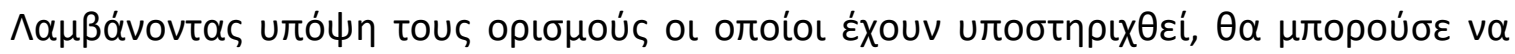

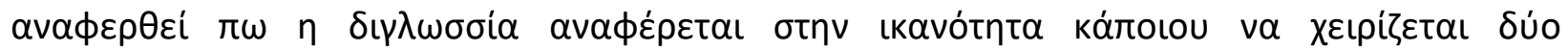

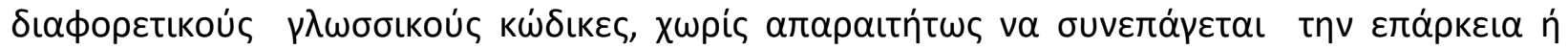

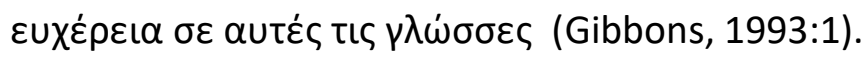

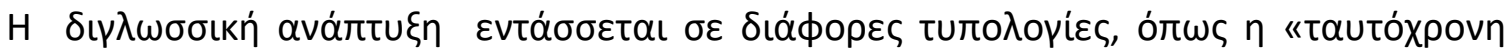

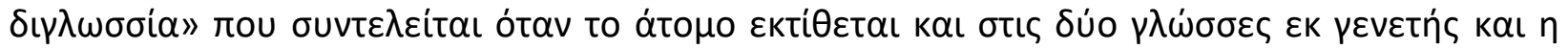

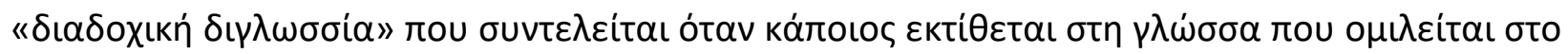

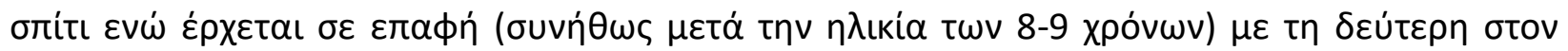

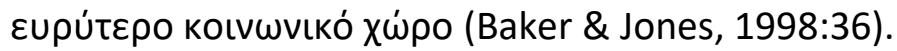

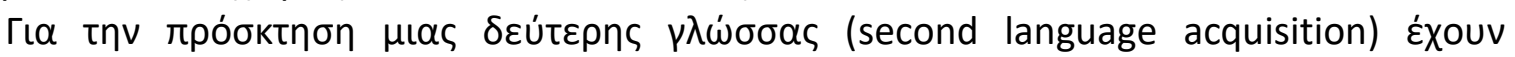

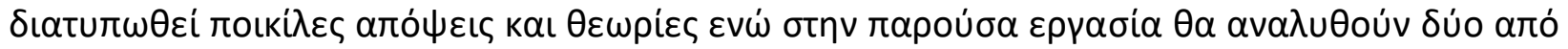

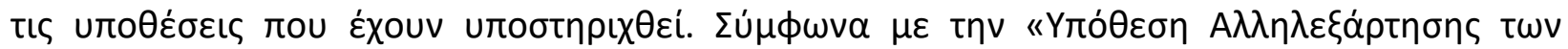

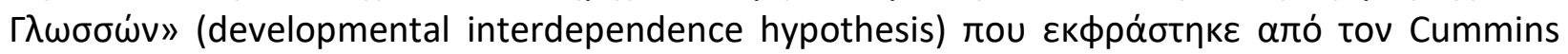

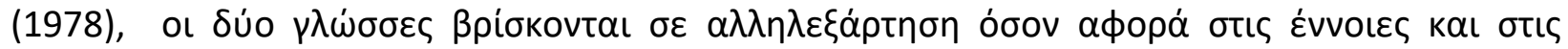

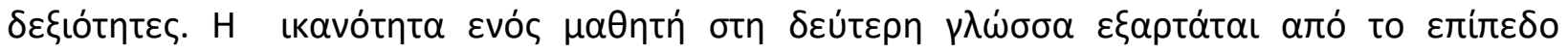

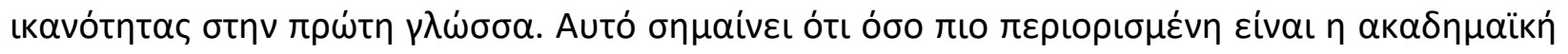

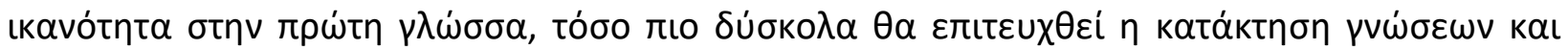

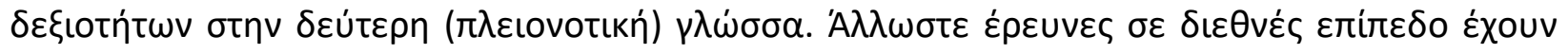


MULTILINGUAL ACADEMIC JOURNAL OF EDUCATION AND SOCIAL SCIENCES

Vol. 5 No. 1, 2017, E-ISSN: 2308-0876 @ 2017 KWP

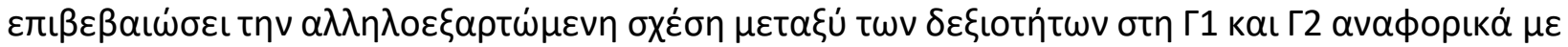

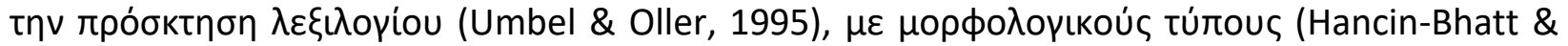

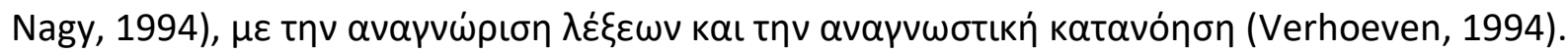

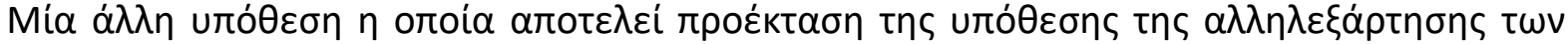

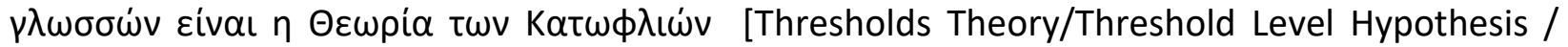

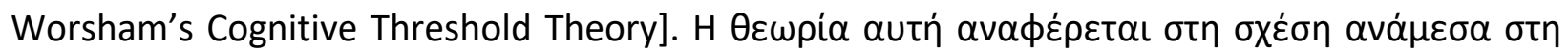

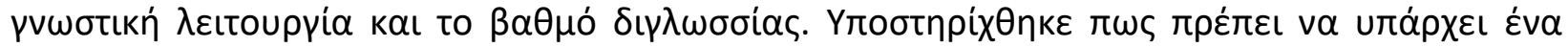

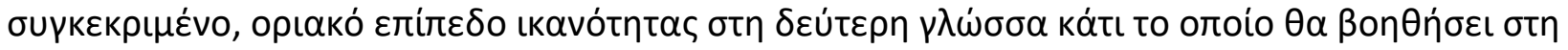

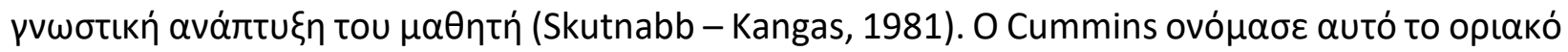

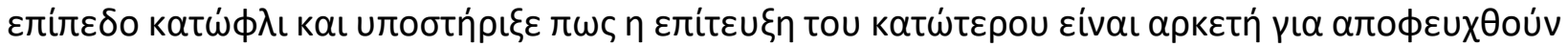

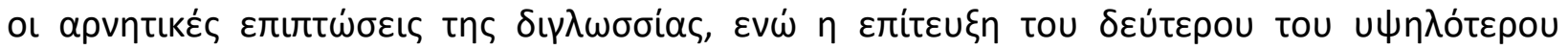

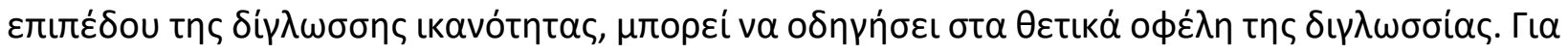

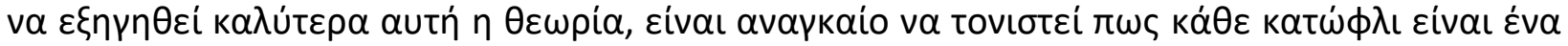

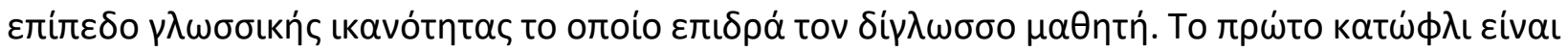

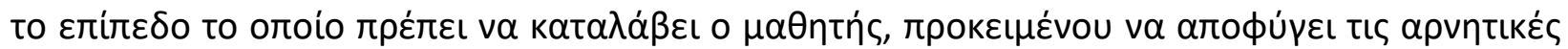

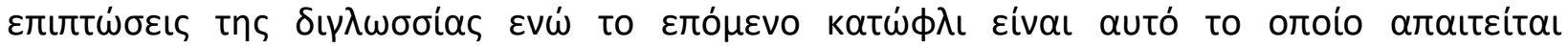

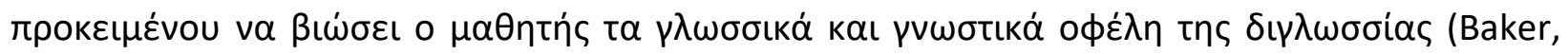
2001).

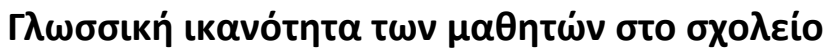

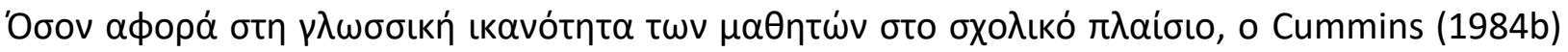

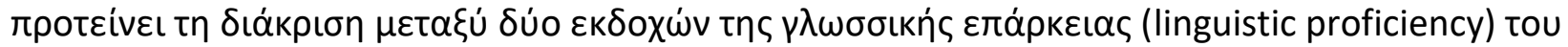

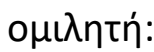

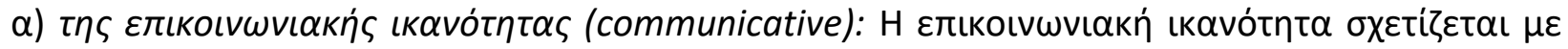

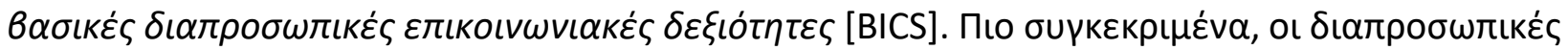

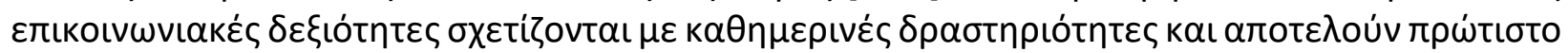

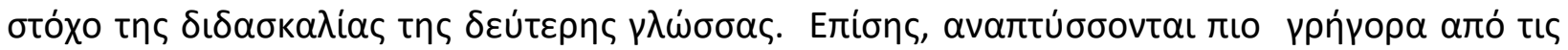

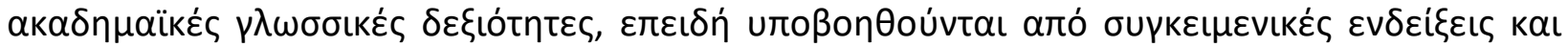

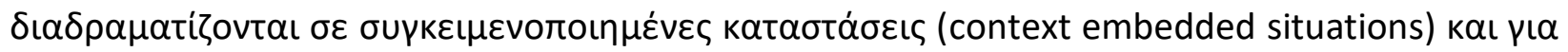

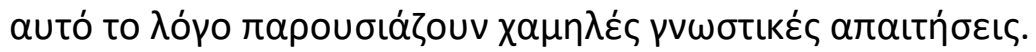

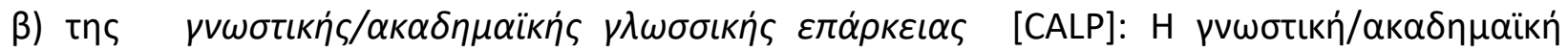

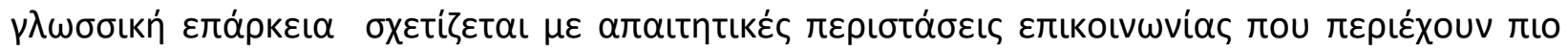

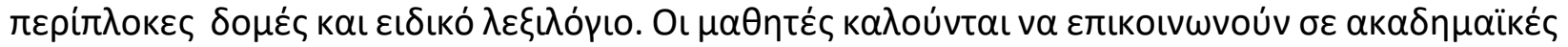

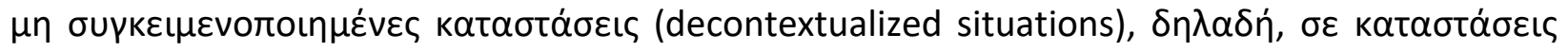

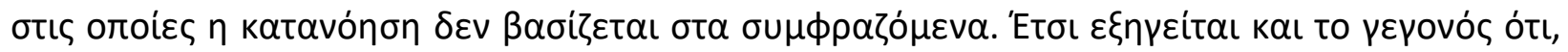

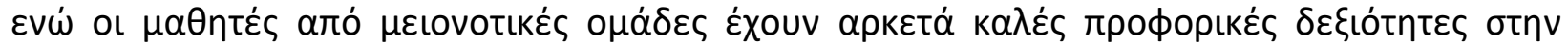

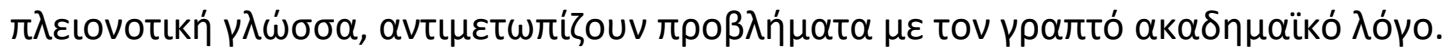

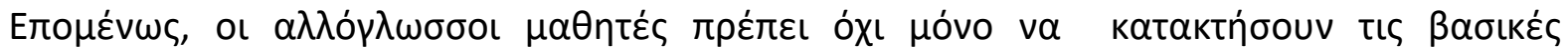

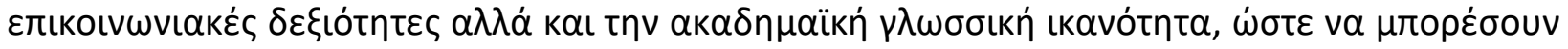

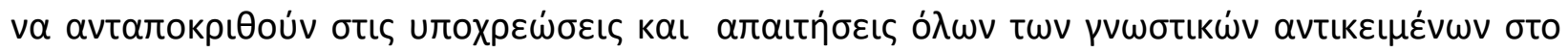
$\sigma \chi 0 \lambda \varepsilon i ́ o$.

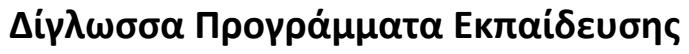

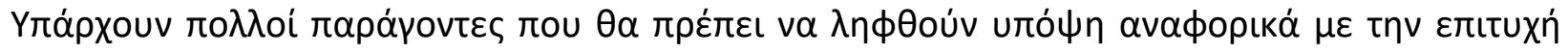


MULTILINGUAL ACADEMIC JOURNAL OF EDUCATION AND SOCIAL SCIENCES

Vol. 5 No. 1, 2017, E-ISSN: 2308-0876 @ 2017 KWP

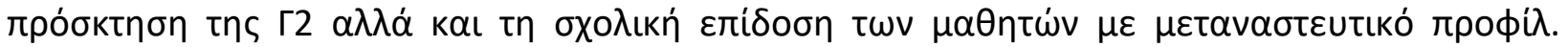

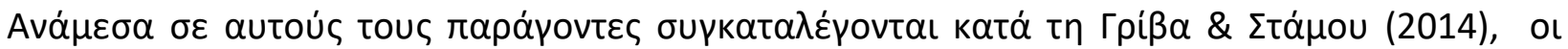

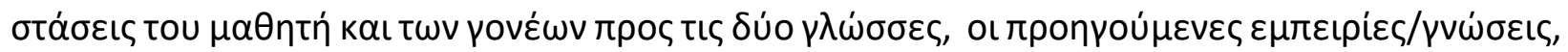

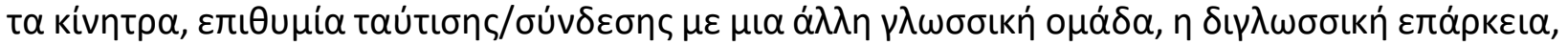

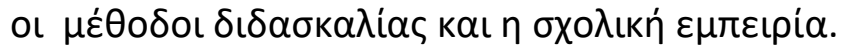

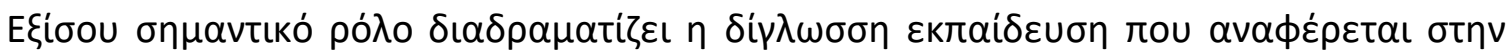

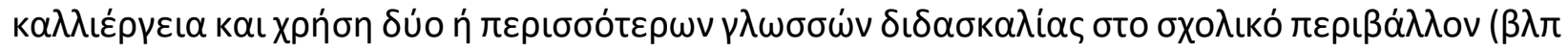

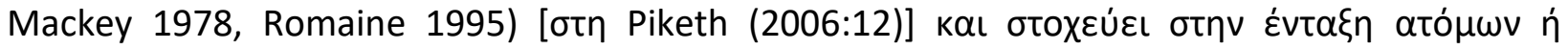

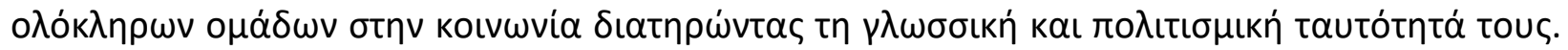

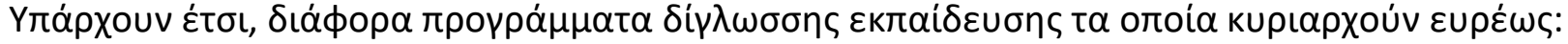

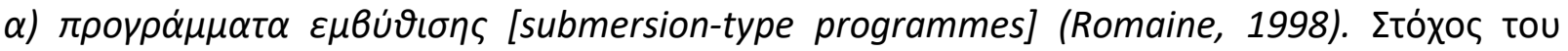

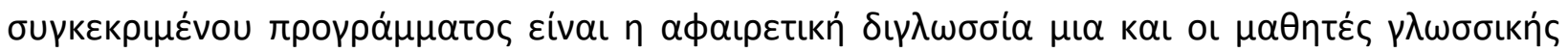

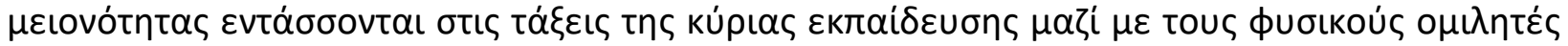

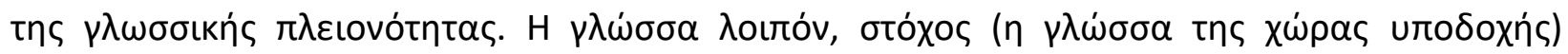

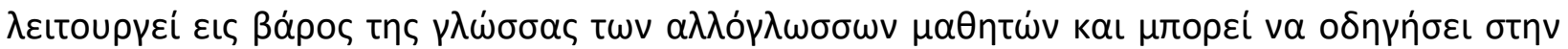

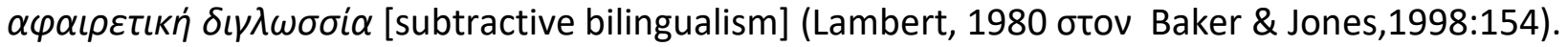

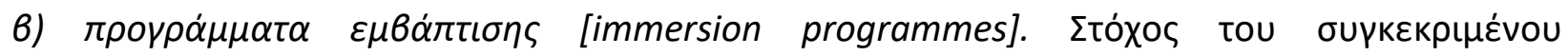

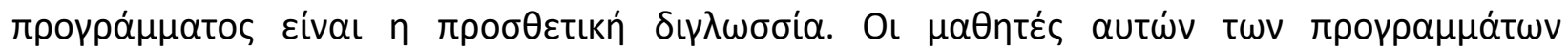

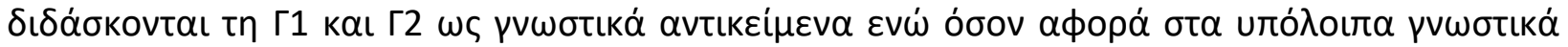

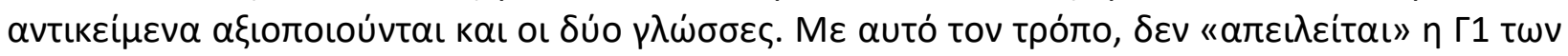

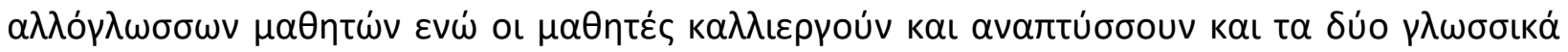

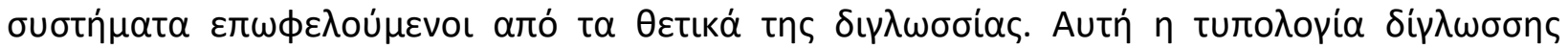

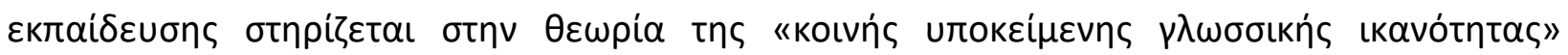

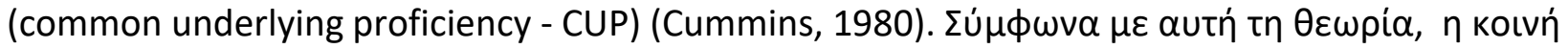

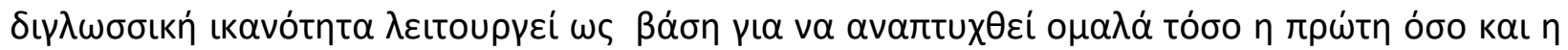

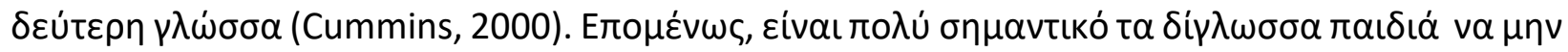

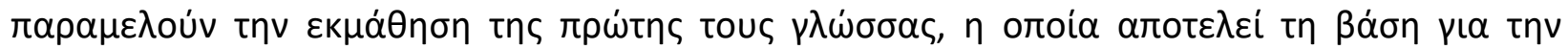

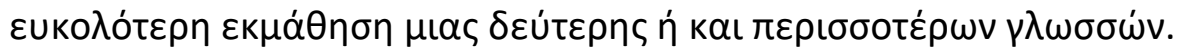

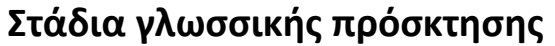

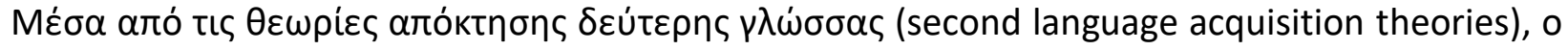

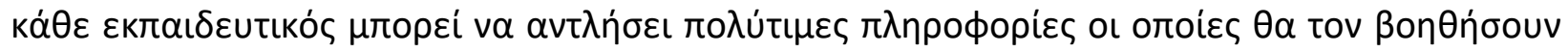

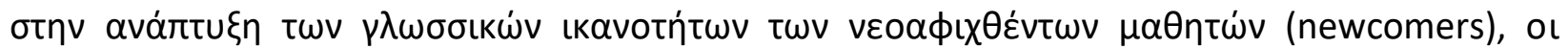

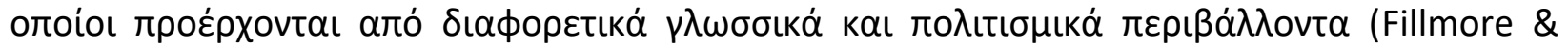

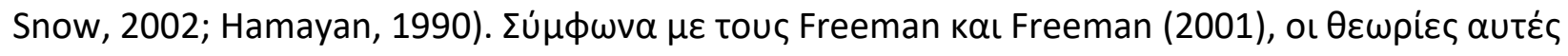

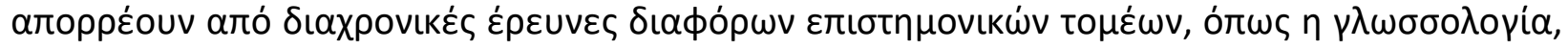

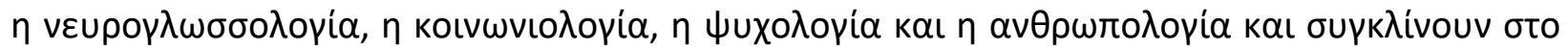

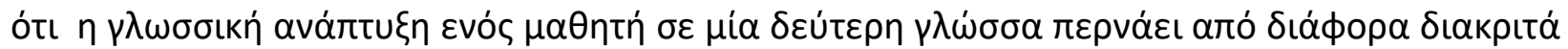

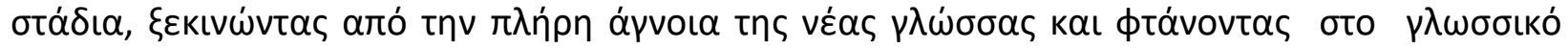

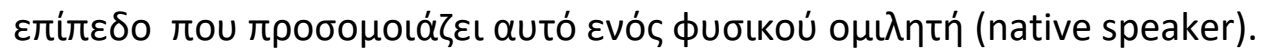

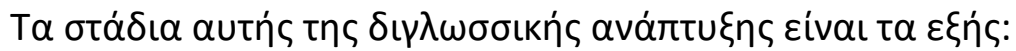

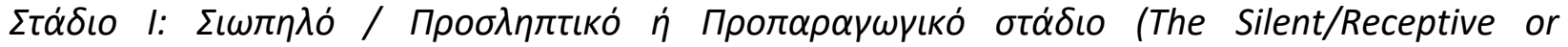
Preproduction Stage)

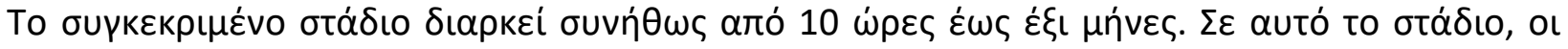


MULTILINGUAL ACADEMIC JOURNAL OF EDUCATION AND SOCIAL SCIENCES

Vol. 5 No. 1, 2017, E-ISSN: 2308-0876 @ 2017 KWP

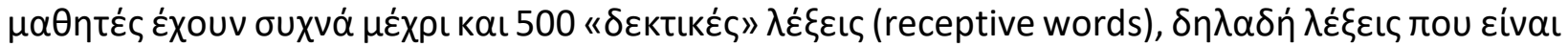

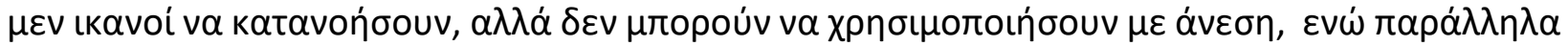

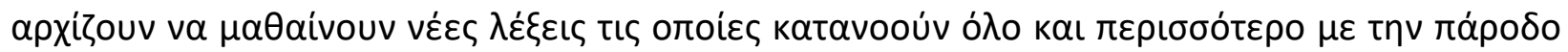
tou xpóvou.

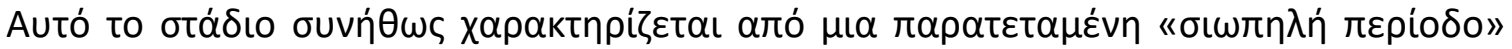

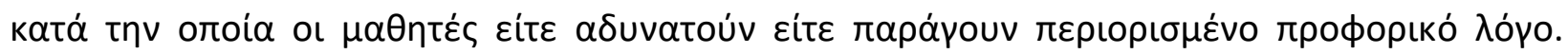

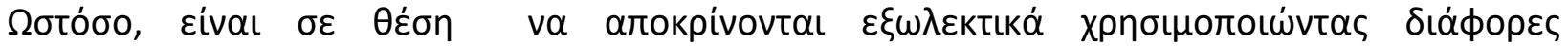

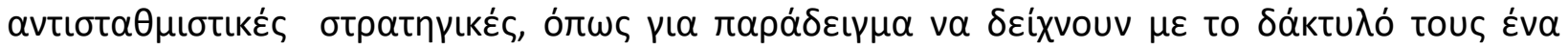

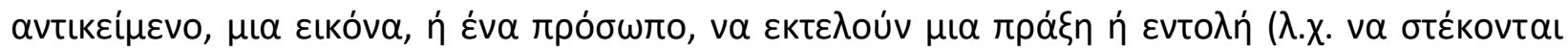

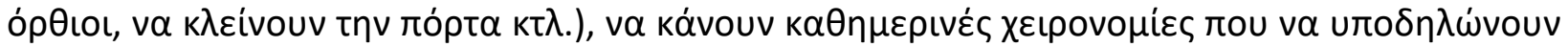

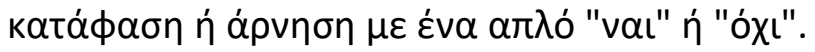

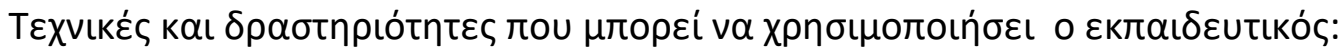

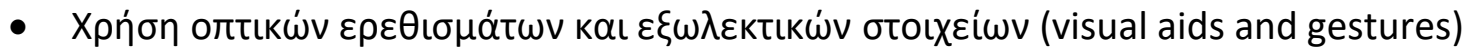

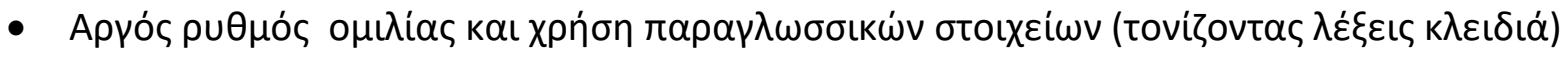

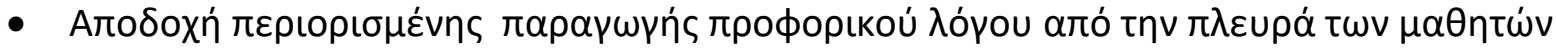

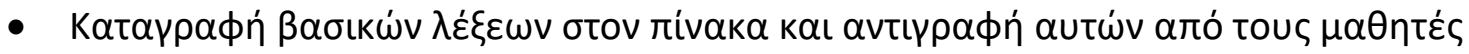

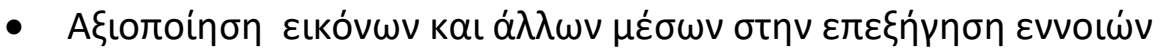

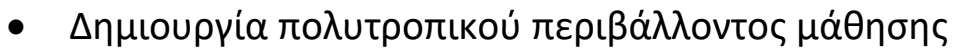

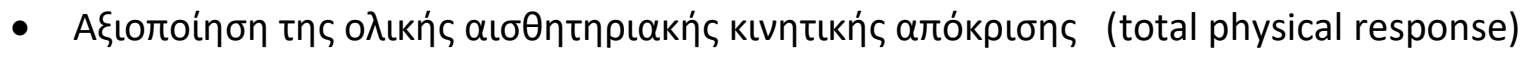

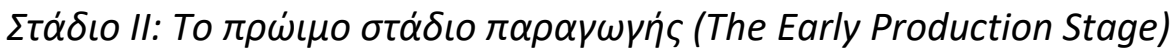

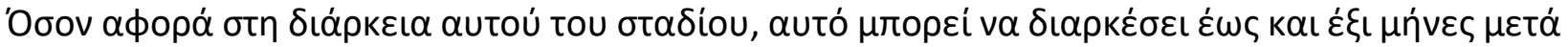

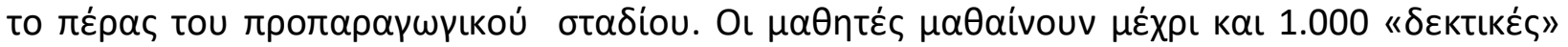

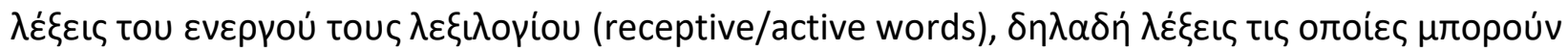

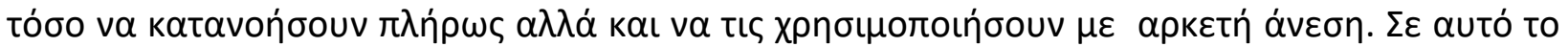

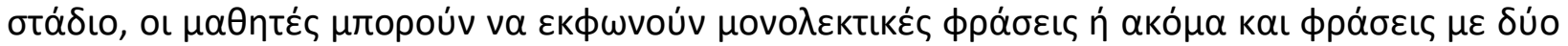

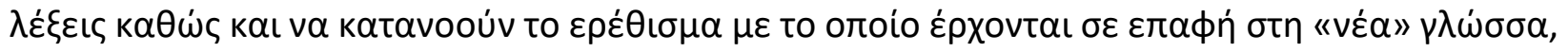

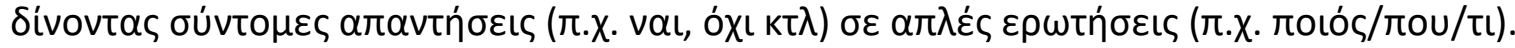

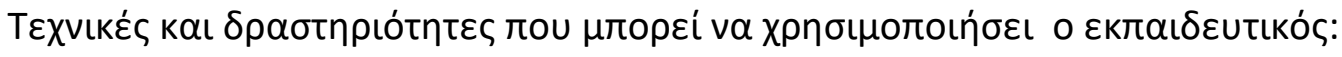

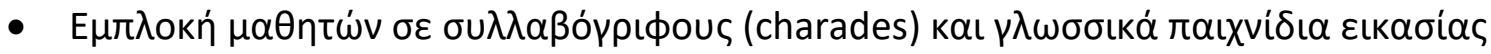

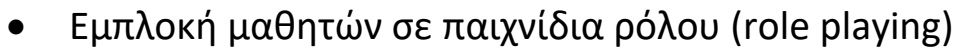

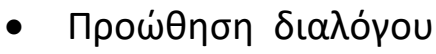

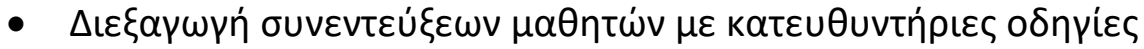

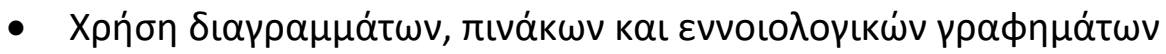

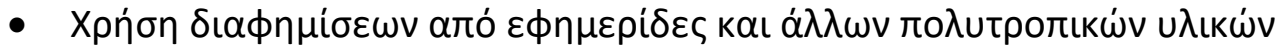

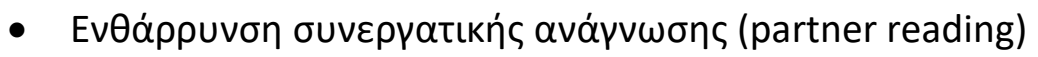

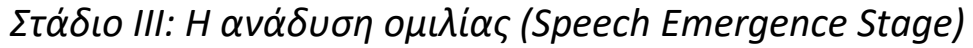

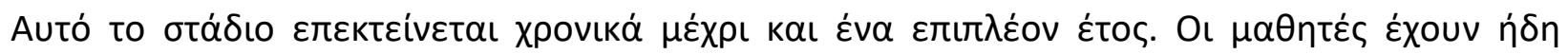

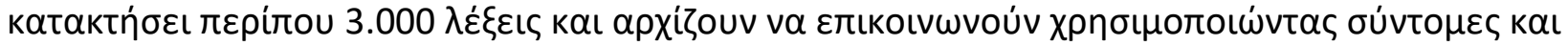

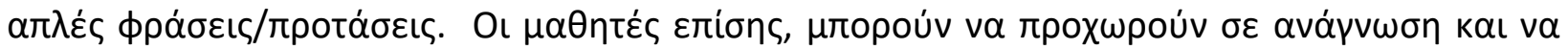

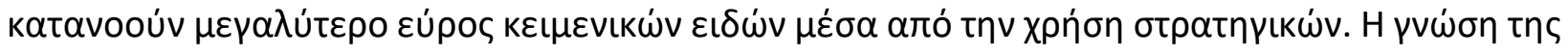

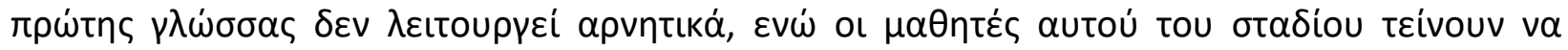


MULTILINGUAL ACADEMIC JOURNAL OF EDUCATION AND SOCIAL SCIENCES

Vol. 5 No. 1, 2017, E-ISSN: 2308-0876 @ 2017 KWP

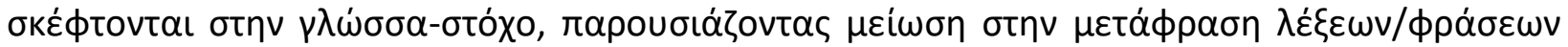

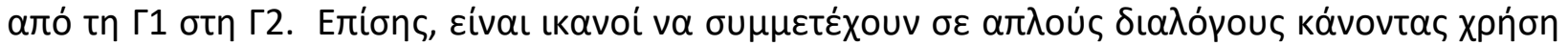

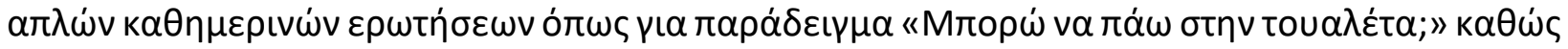

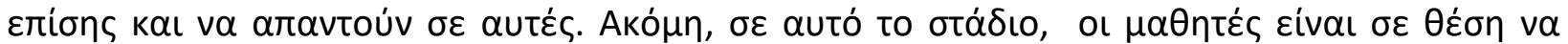

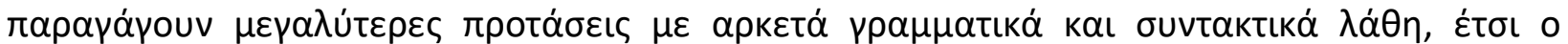

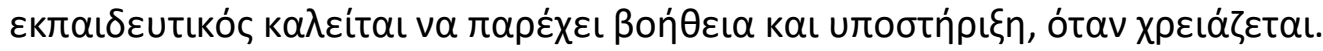

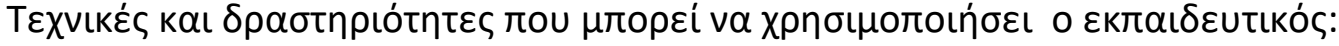

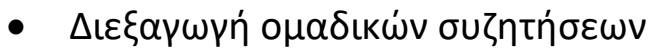

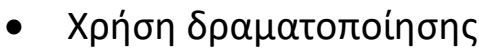

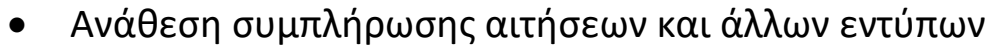

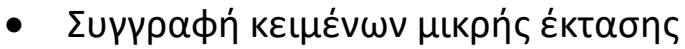

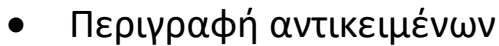

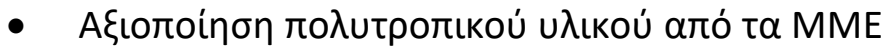

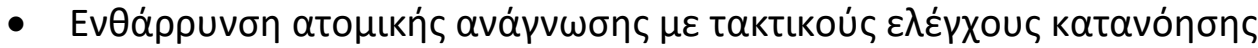

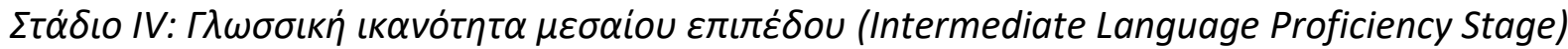

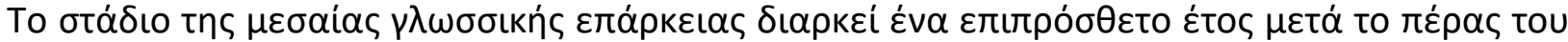

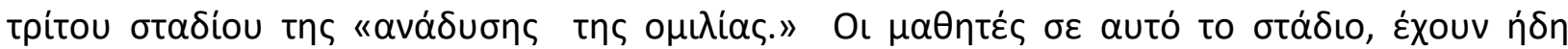

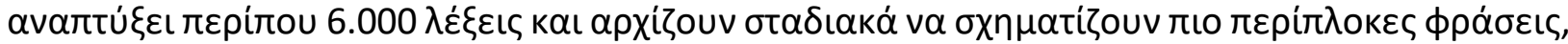

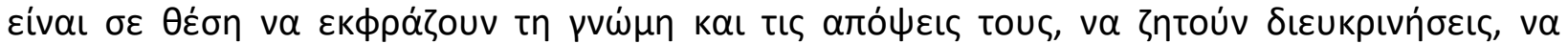

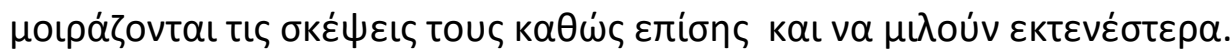

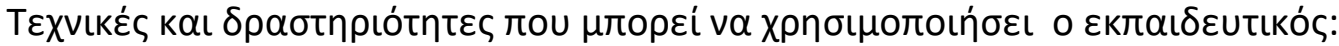

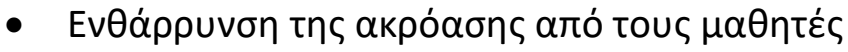

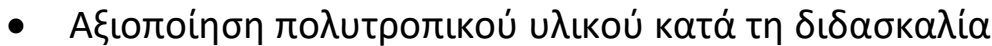

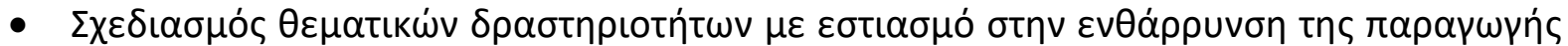

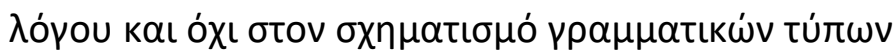

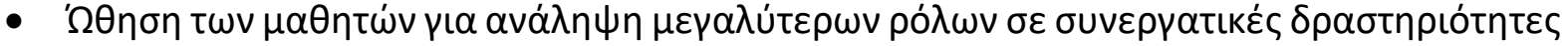

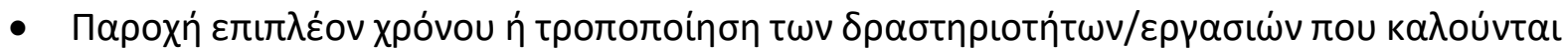

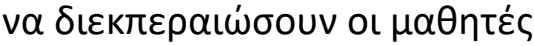

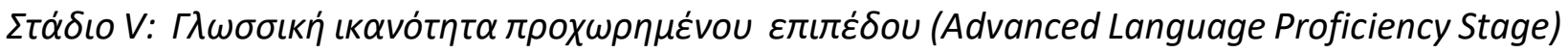

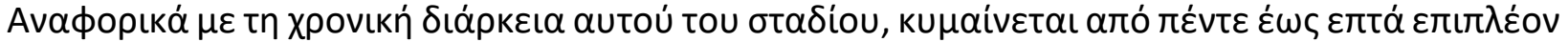

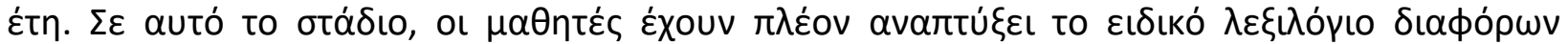

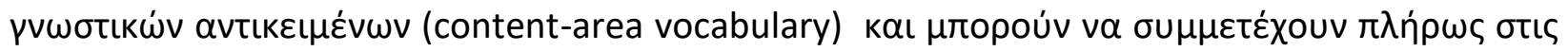

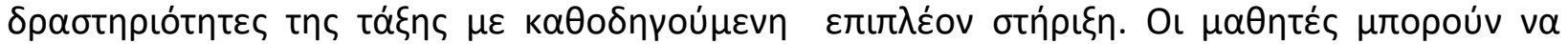

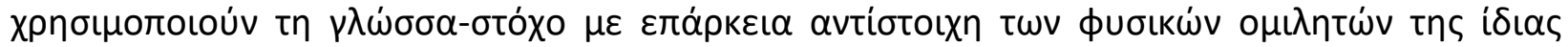

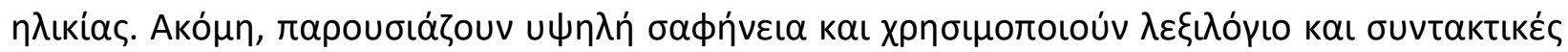

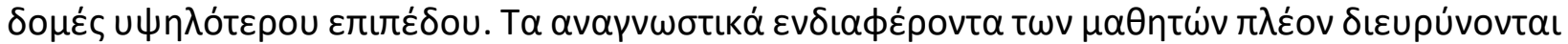

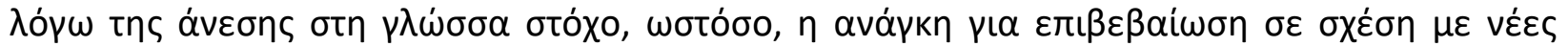

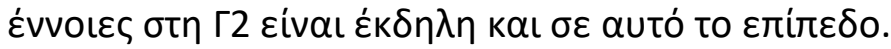

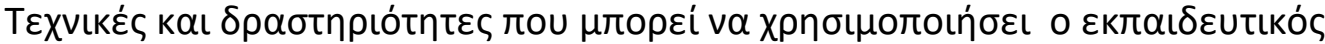

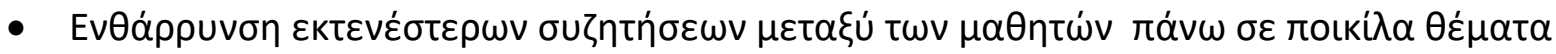

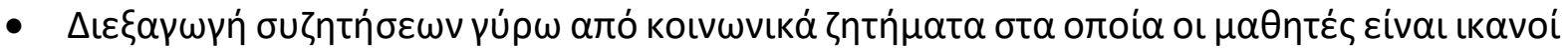


MULTILINGUAL ACADEMIC JOURNAL OF EDUCATION AND SOCIAL SCIENCES

Vol. 5 No. 1, 2017, E-ISSN: 2308-0876 @ 2017 KWP

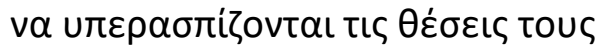

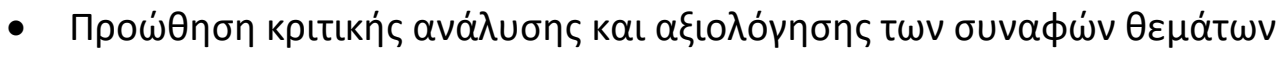

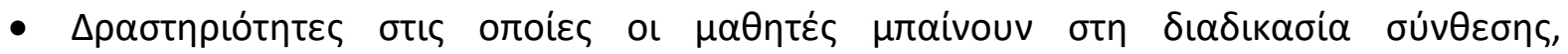

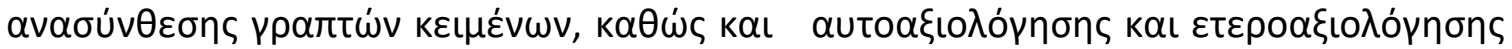

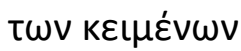

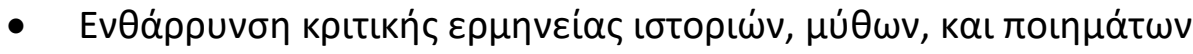

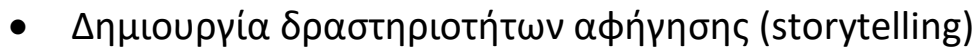

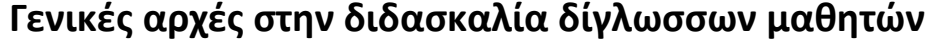

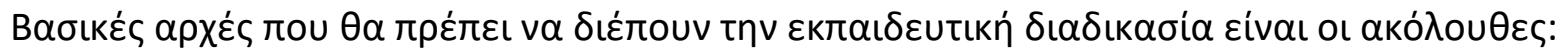

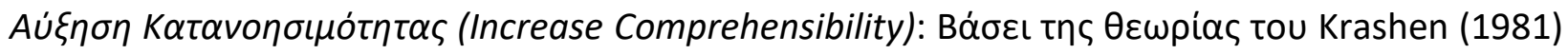

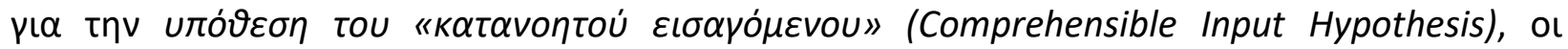

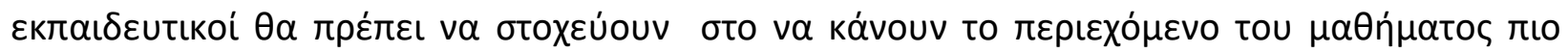

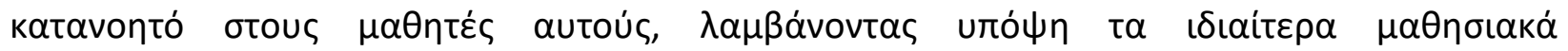

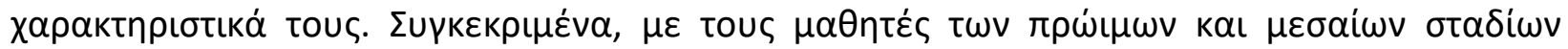

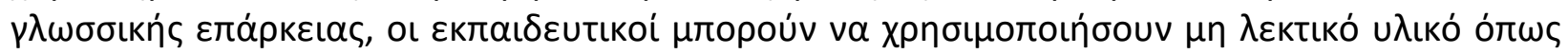

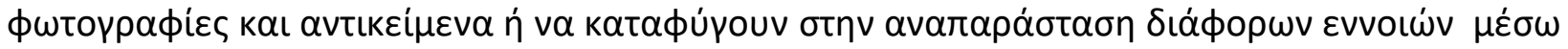

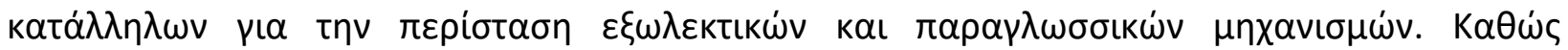

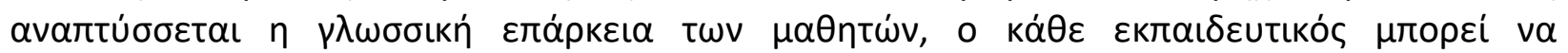

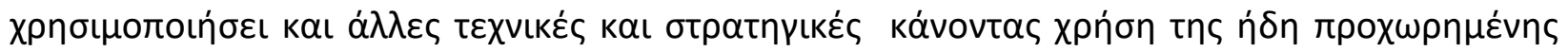

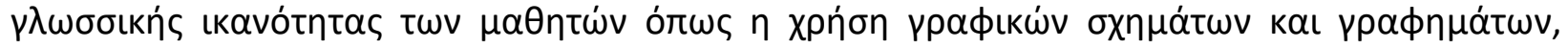

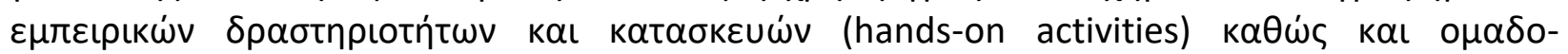

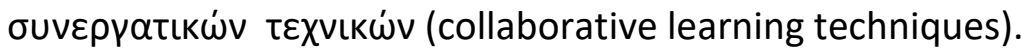

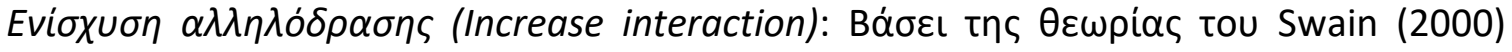

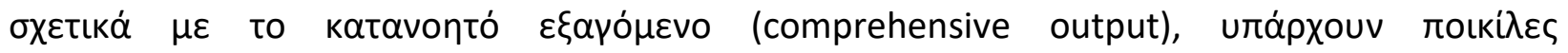

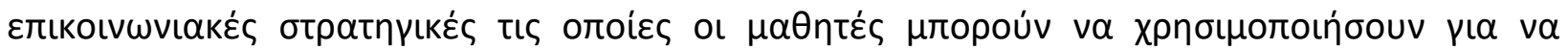

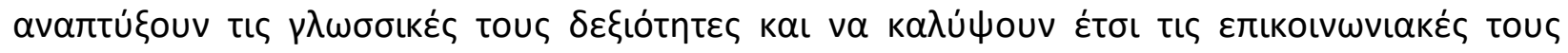

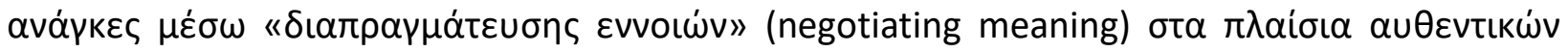

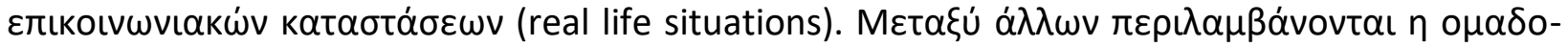

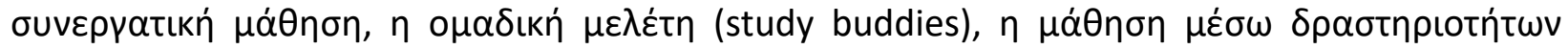

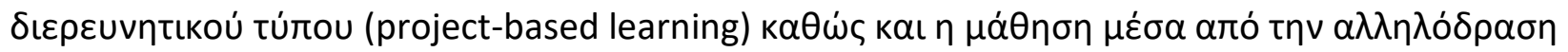

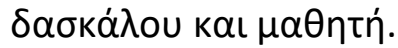

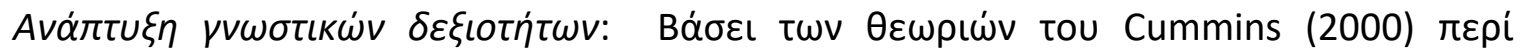

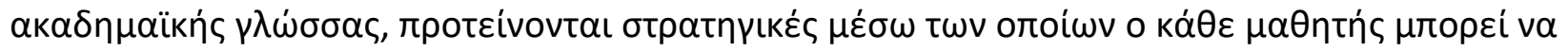

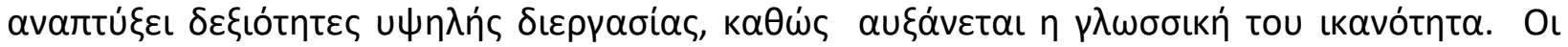

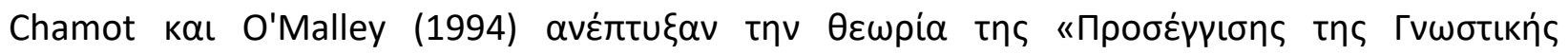

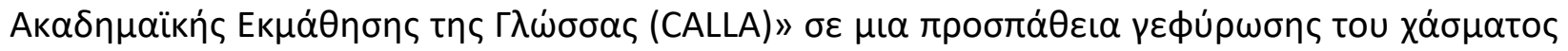

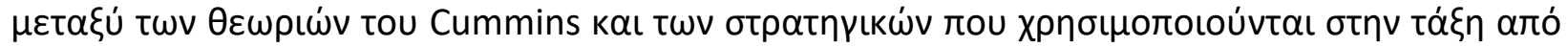

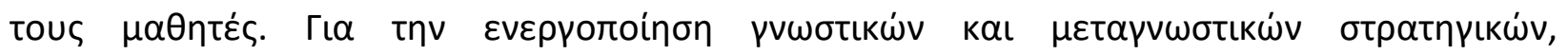

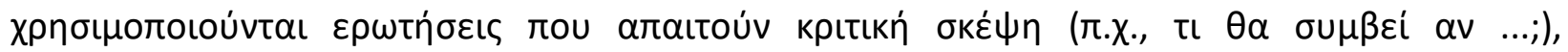

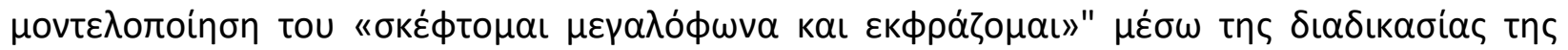

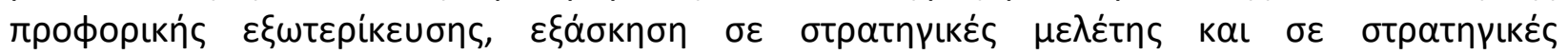

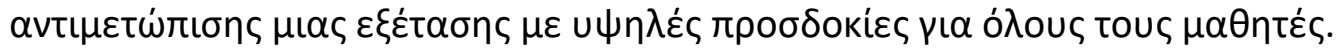

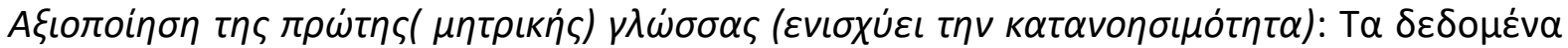


MULTILINGUAL ACADEMIC JOURNAL OF EDUCATION AND SOCIAL SCIENCES

Vol. 5 No. 1, 2017, E-ISSN: 2308-0876 @ 2017 KWP

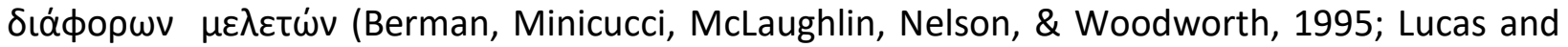

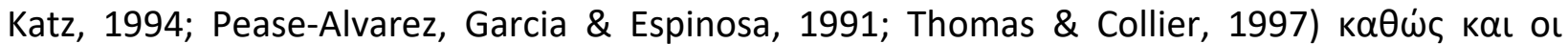

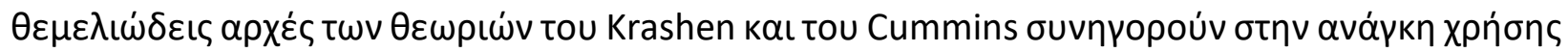

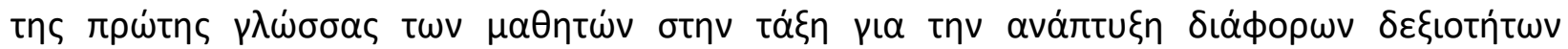

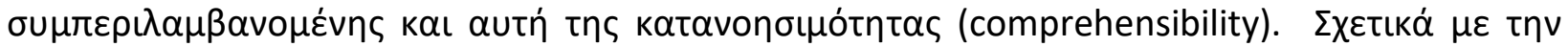

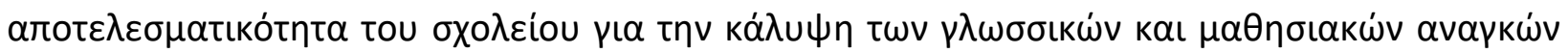

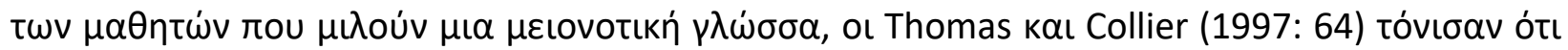

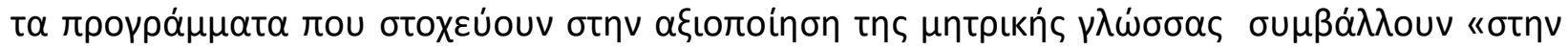

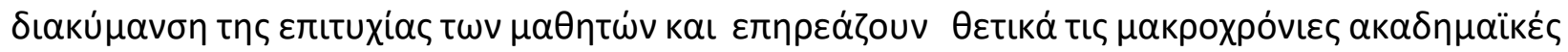

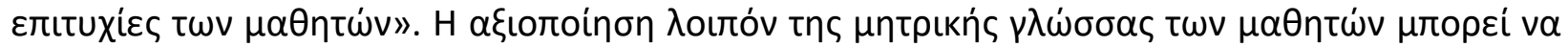

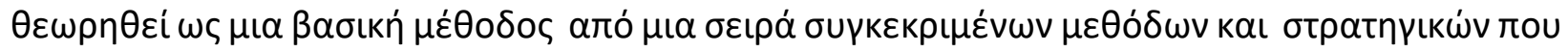

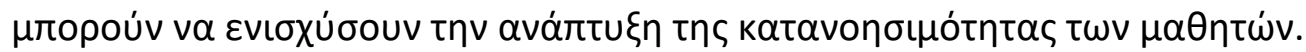

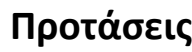

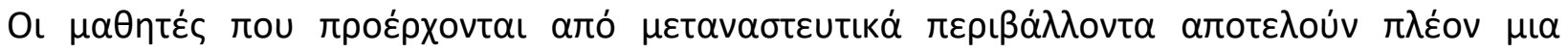

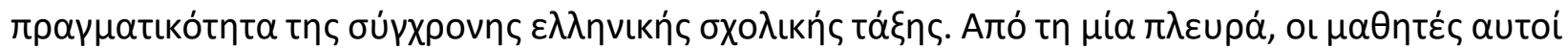

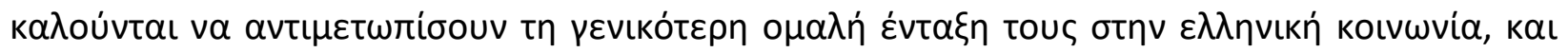

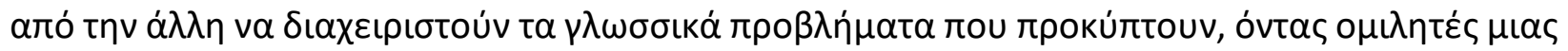

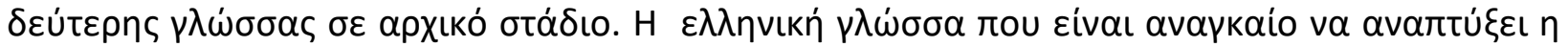

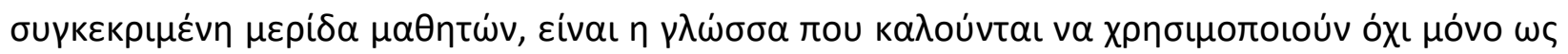

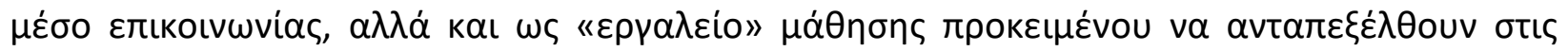

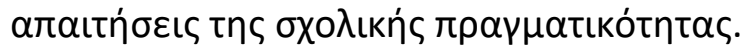

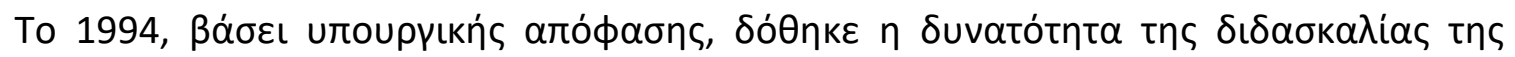

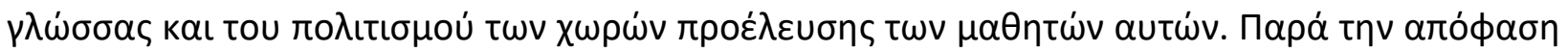

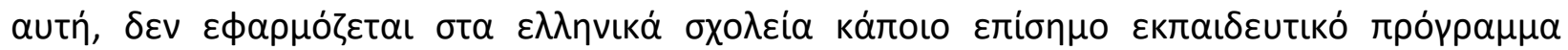

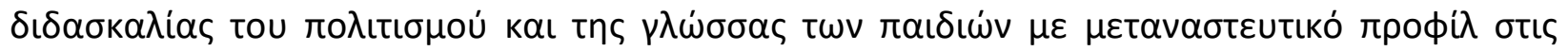

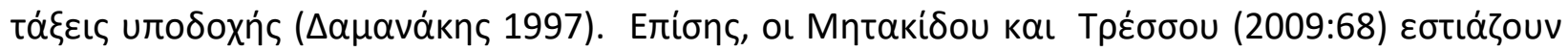

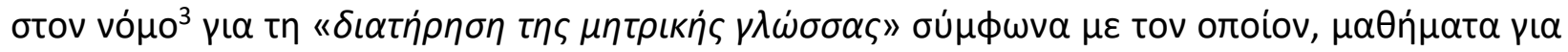

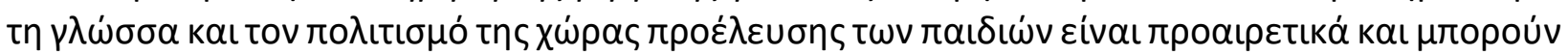

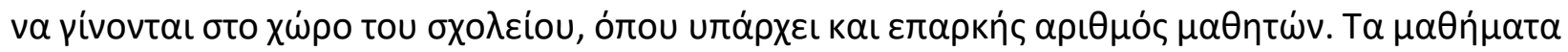

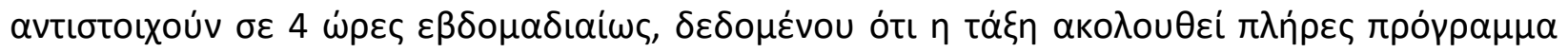

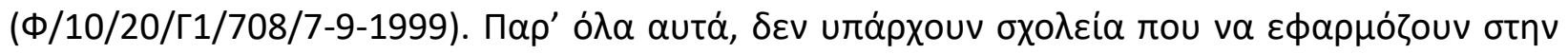

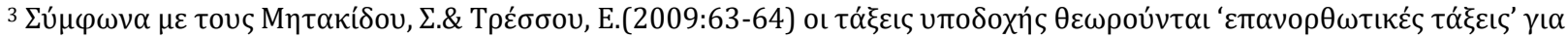

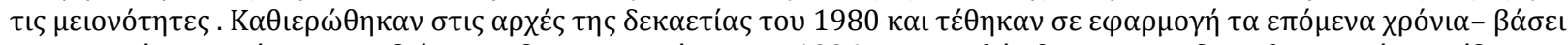

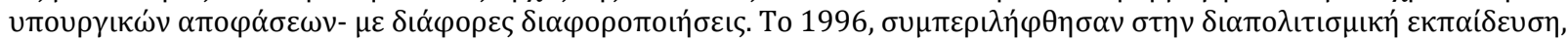

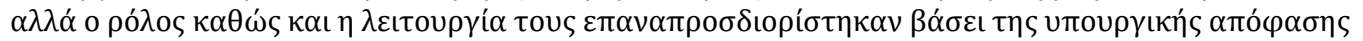

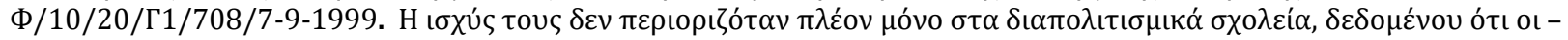

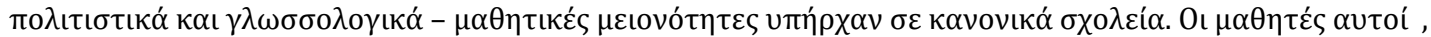

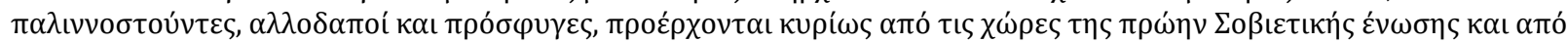

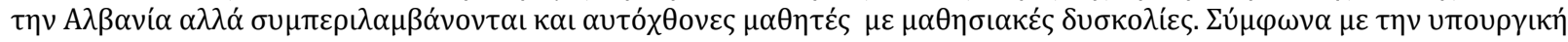

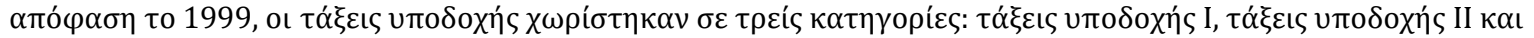

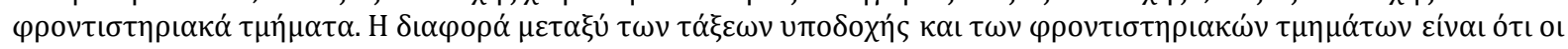

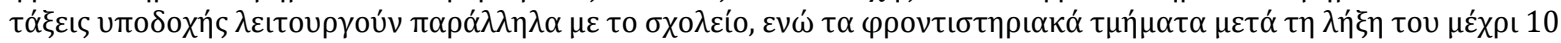

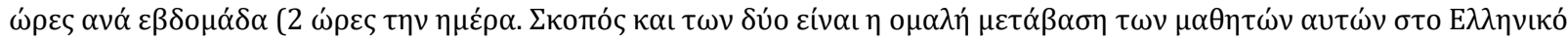

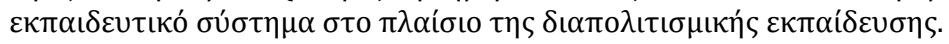


MULTILINGUAL ACADEMIC JOURNAL OF EDUCATION AND SOCIAL SCIENCES

Vol. 5 No. 1, 2017, E-ISSN: 2308-0876 @ 2017 KWP

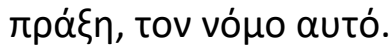

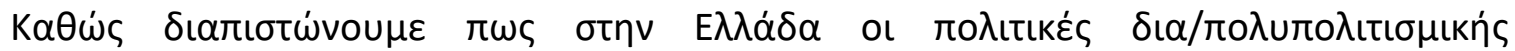

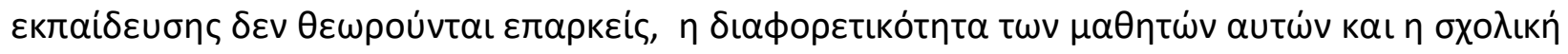

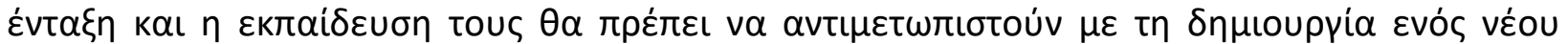

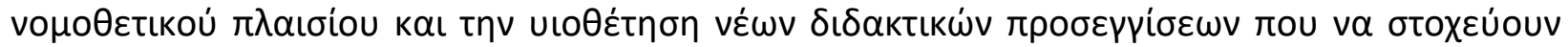

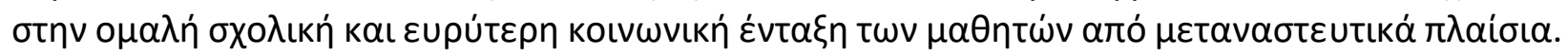

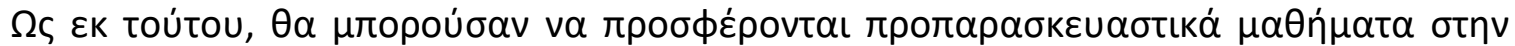

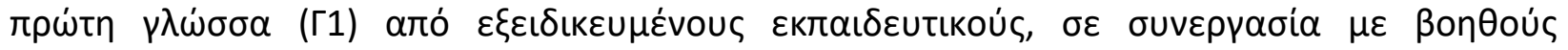

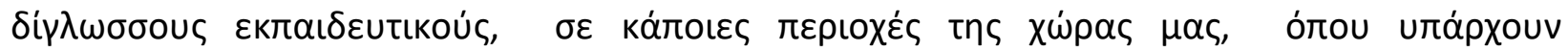

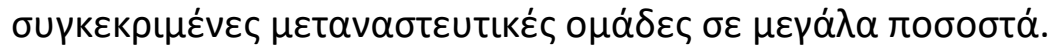

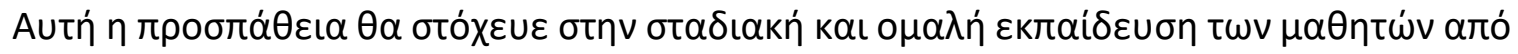

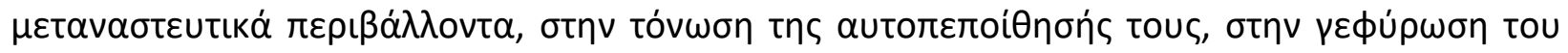

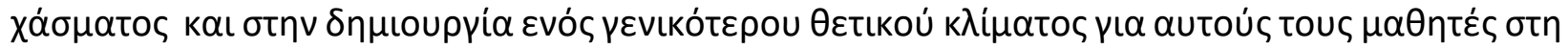

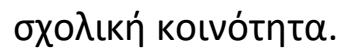

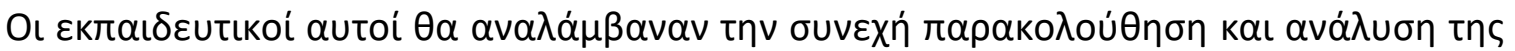

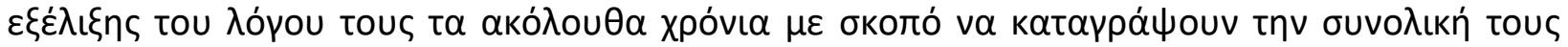

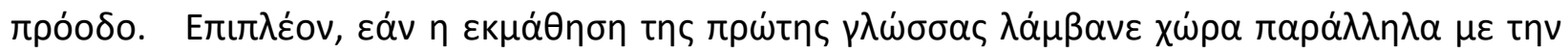

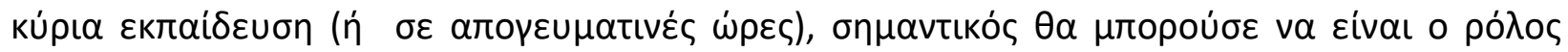

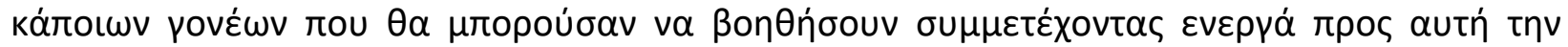

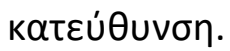

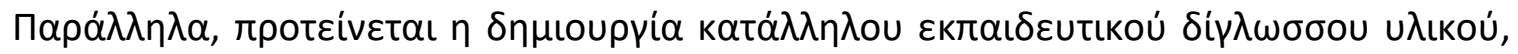

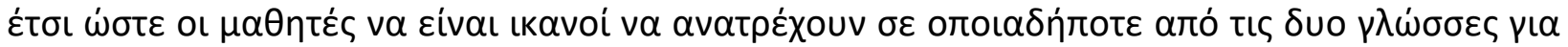

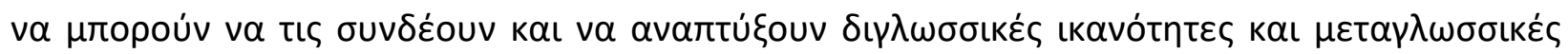

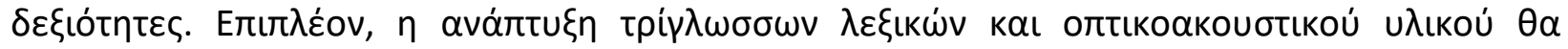

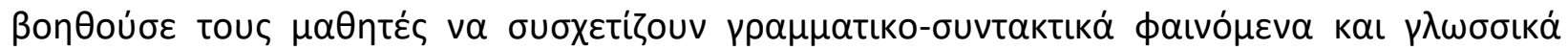

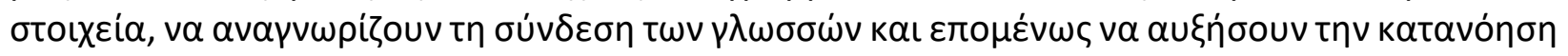

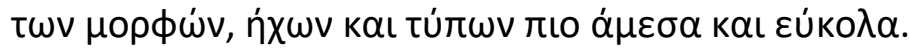

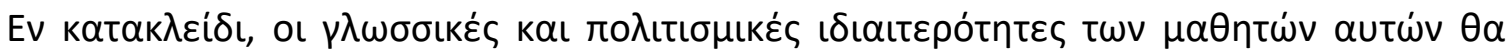

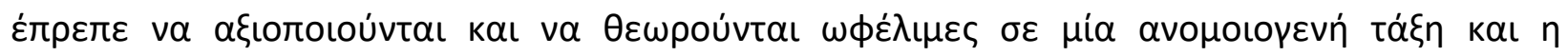

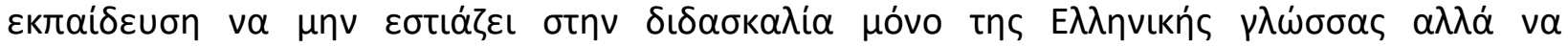

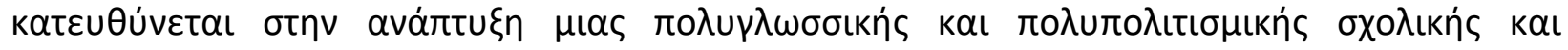

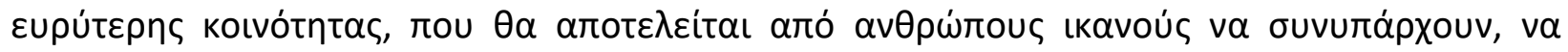

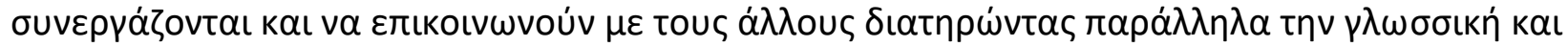

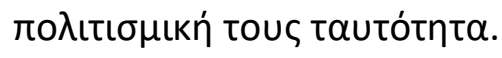

\section{References}

Baker, C., \& Jones, S. P. (1998). Encyclopedia of Bilingualism and Bilingual Education. Clevedon: Multilingual Matters.

Baker, C. (2001). Foundations of bilingual education and bilingualism ( $3^{\text {rd }}$ ed.). Clevedon, UK: Multilingual Matters.

Berman, P., Minicucci, C., McLaughlin, B., Nelson, B., \& Woodworth, K. (1995). School reform and student diversity: Case studies of exemplary practices for LEP students. Washington, DC: National Clearinghouse for Bilingual Education.

Chamot, A. U., \& O'Malley, J. M. (1994). The CALLA handbook: Implementing the cognitive 
MULTILINGUAL ACADEMIC JOURNAL OF EDUCATION AND SOCIAL SCIENCES

Vol. 5 No. 1, 2017, E-ISSN: 2308-0876 @ 2017 KWP

academic language learning approach. Reading, MA: Addison-Wesley.

Cummins, J. (1978). Metalinguistic development of children in bilingual education programmes: data from Irish and Canadian Ukranian - English programmes. In M. Paradis (ed.), Aspects of bilingualism. Columbia: Hornbeam Press.

Cummins, J. (1980). The construct of language proficiency in bilingual education. In J.E. Alatis (ed.) Georgetown University Round Table on Languages and Linguistics. Washington DC: Georgetown University Press.

Cummins, J. (1984). Bilingualism and Cognitive functioning. In S.Shapson \&V. Douley (eds), Bilingual and multicultural education: Canadian Perspectives. Clevedon: Multilingual Matters.

Cummins, J. (2000). Language, Power and Pedgogy: Bilingual Children in the Crossfire. Clevedon: Multilingual Matters.

Edwards, J. (2003). The importance of being bilingual. In J. M. Dewaele, A. Housen, \& L.Wei (Eds.), Bilingualism: Beyond basic principles (pp. 28-42). New York: Multilingual Matters.

Fillmore, L. W., \& Snow, C. (2002). What teachers need to know about language. In C. T. Adger, C. Snow, \& D. Christian (Eds.), What teachers need to know about language (pp. 7-53). Washington, DC: Center for Applied Linguistics.

Freeman, D., \& Freeman, Y. (1991). Practicing what we preach: Whole language with teachers of bilingual learners. In K. Goodman \& Y. Goodman (Eds.), Occasional papers: Program in language and literacy. Tucson, AZ: University of Arizona.

Gibbons, P. (1993). Learning to learn in a second language. Portsmouth, NH: Heinemann.

Griva, E., \& Chostelidou, D. (2012), Additive bilingualism of immigrant children: Introducing a multisensory project in kindergarten. In H. Switzer D. Foulker (Eds), Kindergartens: Teaching Methods, Expectations and Current Challenges, 83-104. Nova Science publishers Inc.

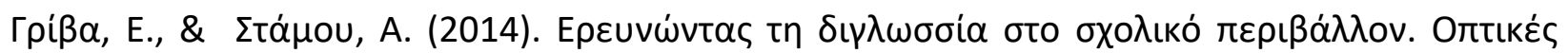

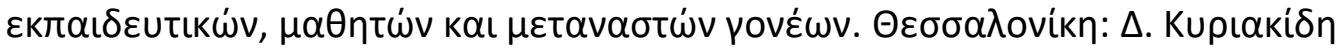

Hamayan, E. V. (1990). Preparing mainstream classroom teachers to teach potentially English proficient students. In Proceedings of the First Research Symposium on Limited English Proficient Students' Issues (pp. 1-21). Washington, DC: U.S. Department of Education, Office of Bilingual Education and Minority Language Affairs.

Hancen-Bhatt, B., \& Nagy, W. (1994). Lexical transfer and second language morphological development. Applied Psycholinguistics, 15, 289-310.

Haugen, E. (1953). The Norwegian Language in America: A study in Bilingual behavior. Philadelphia: University of Pennsylvania Press.

Karosas, S. (2004). Bilingualism in Theory and Practice. URL: www.svenskamammor.com/uppsats.htm.

Krashen, S. D. (1981). Second language acquisition and second language learning. New York, NY: Pergamon Press.

Lucas, T., \& Katz, A. (1994). Reframing the debate: The roles of native languages in English-only programs for language minority students. TESOL Quarterly, 28(3), 537-561.

Ludi, G. (1986). Forms and functions of bilingual speech in pluricultural migrant communities in Switzerland. In J.A. Fishman, A. Tabouret-Keller, M. Clyne, B. Krisbnamurti \& M. H. Abdulaziz (Eds.), The Fergusonian Impact. Vol. 2: Sociolinguistics and the Sociology of 
MULTILINGUAL ACADEMIC JOURNAL OF EDUCATION AND SOCIAL SCIENCES

Vol. 5 No. 1, 2017, E-ISSN: 2308-0876 @ 2017 KWP

Language, 217-236, Berlin, New York and Amsterdam: Mouton de Gruyter.

Mitakidou, S., \& Tressou, E. (2009). Cross-Cultural Education: A Challenge or a Problem? International Critical Childhood Policy Studies 2/1: 61-74.

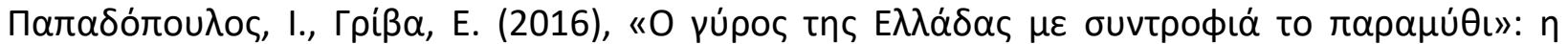

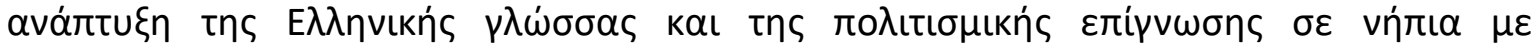

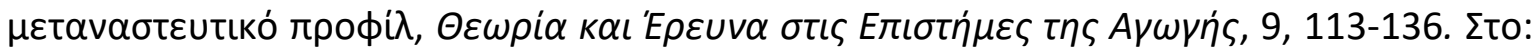
http://periodiko.inpatra.gr.

Pease-Alvarez, L., Garcia, E., \& Espinosa, P. (1991). Effective instruction for language minority students: An early childhood case study. Early Childhood Research Quarterly, 6(3), 347363.

Piketh, A. P. M. (2006). Dutch Bilingual Education: a new phenomenon or just the same old song? A study using bilingual typologies to determine the nature of Dutch Bilingual Education. Dissertation. Oxford Brookes University.

Romaine, S. (1995). Bilingualism. Oxford, Blackwell.

Skutnabb-Kangas, T. (1984). Bilingualism or not: The Education of Minorities. Clevedon: Multilingual Matters.

Swain, M. (2000). The output hypothesis and beyond: Mediating acquisition through collaborative dialogue. In J.P. Lantolf (Ed.), Sociocultural theory and second language learning (pp. 97-114). New York, NY: Oxford University Press.

Thomas, W. P., \& Collier, V. (1997). School effectiveness for language minority students. (NCBE Resource Collection Series No. 9). Washington, DC: National Clearinghouse for Bilingual Education.

Umbel, V. M., and Kimbrough, O. (1995). Developmental Changes in Receptive Vocabulary in Hispanic Bilingual School Children. In Lexical Issues in language learning (pp. 59-80). Amsterdam: John Benjamins.

Verhoeven, L. (1994). Transfer in bilingual development: The linguistic interdependency hypothesis revisited. Language Learning, 44, 381-415. 